\title{
A NEW SAMPLE OF FAINT HALO B STARS
}

\author{
KENNETH J. MITCHELL \\ General Sciences Corporation \\ 6100 Chevey Chase Dr., Laurel, MD 20707 \\ REX A. SAFFER \\ Space Telescope Science Institute \\ 3700 San Martin Dr., Baltimore, MD 21218
}

AND

STEVE B. HOWELL

Planetary Science Institute

2421 E. 6th St., Tucson, AZ 85719

\section{Summary}

This poster reports the isolation of a new, complete sample of 24 faint halo B stars. These B stars were selected as blue- and ultraviolet-excess objects in the US survey at high Galactic latitudes (Usher and Mitchell 1990), and were given preliminary classifications using low-resolution spectrophotometry. The new sample is complete over 206 square degrees of sky to faint magnitude completeness limits in the range $B=16.5$ and $B=18.3$.

Stromgren color indices for the US B stars have been derived from the spectrophotometry through numerically convolved filters (Howell 1986). The colors have been used to help define the red completeness limit of the sample at $(b-y)=-0.01$, at the B9.5/A0 classification boundary. In addition, surface gravity and temperature estimates useful for separating the hotter B stars from sdB stars have been provided by atmospheric model fitting to the existing spectra (cf. Saffer et al. 1994).

\section{References}

Howell, S.B. (1986) Astronomical Journal, 91, 171.

Saffer, R.A. et al. (1994) Astrophysical Journal, 432, 351.

Usher, P.D. and Mitchell, K.J. (1990) Astrophysical Journal Supplement, 74, 885. 